\title{
Surgical treatment of haemorrhoidal disease - the current situation in Poland
}

\author{
Lukasz Dziki, Michal Mik, Radzislaw Trzcinski, Jaroslaw Buczynski, Anna Kreisel, Mariusz Skoneczny, Adam Dziki \\ Department of General and Colorectal Surgery, Medical University of Lodz, Lodz, Poland
}

Gastroenterology Rev 2016; 11 (2): 111-114

DOI: $10.5114 / p g .2016 .57616$

Key words: haemorrhoids, surgery, treatment.

Address for correspondence: Michal Mik MD, PhD, Department of General and Colorectal Surgery, Medical University of Lodz, Plac Hallera 1, 90-647 Lodz, Poland, phone: +48 504048 127, fax: +48 4263930 76, e-mail: m.mik@wp.pl

\begin{abstract}
Introduction: In cases of haemorrhoidal disease resistant to conservative treatment, surgical treatment is necessary to relieve the symptoms.

Aim: To investigate the current methods used by Polish surgeons.

Material and methods: Surveys were distributed to members of the Association of Polish Surgeons (APS), in which participants were asked a number of closed-ended questions regarding haemorrhoidal disease and the way they treated suffering patients

Results: Out of the 1523 members of APS who received questionnaires, responses were received from 807 (52.9\%) members. The Milligan-Morgan technique was indicated by $72.5 \%$ of surgeons as a leading surgical treatment, followed by Ligasure (15.5\%), Ferguson (3.5\%), DGHL (3.5\%), other methods (3.5\%), Parks (1.7\%), and Longo (0\%). The majority of participants (93\%) indicated from 0 to 1 life threatening complications, $5 \%$ - from 2 to 3, and $2 \%>4$. A total of $83 \%$ of participants use a single dose of antibiotics prior to surgery.

Conclusions: The Milligan-Morgan technique is the preferred method. The majority of procedures are performed in regional hospitals and university departments, and less commonly in private practices. The vast majority of surgeons in Poland are not convinced about the stapler technique, justifying this fact with the possibility of developing serious complications
\end{abstract}

\section{Introduction}

The term haemorrhoids often refers to the pathological symptoms of pain, itching, bleeding, or other palpable abnormalities in the perianal region. Haemorrhoids are normal anatomical structures that have their own physiological function. Patients experiencing the symptoms of haemorrhoidal disease often seek methods of treatment involving permanent removal of haemorrhoids [1, 2].

Haemorrhoidal cushions are structures formed by venous plexus clusters, located in the anal canal, right above the dentate line. Functionally they play a supporting role in aiding the anal sphincter - they are responsible for about $15 \%$ of stool continence ability. States associated with pathological changes of haemorrhoids are known as haemorrhoidal disease $[3,4]$.

Haemorrhoidal disease is a frequent ailment with no sex predominance. It occurs in about $5 \%$ of the population, most commonly between the ages of 45 and 65 years [5]. Aetiological factors associated with haemorrhoidal disease include constipation, diarrhoea, increased and chronic physical straining, pregnancy, increased intra-abdominal pressure associated with decreased venous return, aging, anomalies and dysfunctions of the internal sphincter muscle, and genetic predispositions $[6,7]$.

Symptoms of haemorrhoidal disease include: bright red bleeding from the anal canal, pain in the perianal region, and constant anal prolapse (in the $4^{\text {th }}$ clinical stage of the disease). Other symptoms include difficulty in maintaining hygiene in the perianal and perineal region, cosmetic deformities, as well as itching and burning sensations in the anal region. The control of symptoms should be the priority of the therapy. Symptoms often disappear when conservative therapy has been administered. In patients with symptomatic haemorrhoidal disease, resistant to conservative 
treatment, surgical treatment is necessary to relieve the symptoms [7].

In the past few years, many different surgical methods have been introduced for the treatment of patients with symptomatic haemorrhoidal disease. In available literature, the effectiveness of those treatments shows various results [8].

\section{Aim}

The aim of this study was to investigate currently used methods, to describe the trends and preferences in the surgical treatment of patients with haemorrhoidal disease in Poland, as well as to attempt to set "sort of guidelines" for the surgical treatment of haemorrhoidal disease.

\section{Material and methods}

Surveys were distributed to members of the Association of Polish Surgeons (APS) in which participants were asked a number of closed-ended questions regarding haemorrhoidal disease and the way they treated suffering patients.

After the analysis of questionnaires, general conclusions were made about the surgical treatment of haemorrhoidal disease in Poland.

Participants were asked to answer questions concerning the following topics: the preferred setting for the treatment of haemorrhoidal disease; the annual number of patients treated conservatively and those treated surgically for haemorrhoidal disease; and the preferred surgical method for the treatment of haemorrhoidal disease, giving them the following options to choose from: Milligan-Morgan method, Ferguson method, Longo method, Parks method, Doppler guided hemorrhoidal artery ligation (DGHL), other methods; as well as the percentage of recurrences and serious, life-threatening complications following each of the methods of treatment. In addition, participants were also asked to state what they considered to be the least patient-friendly method from the ones mentioned above. Participants were also asked to estimate the percentage of procedures in which the stapler method was used, and another question was to establish the reason for the infrequent use of the Longo method. In addition, participants were asked to indicate a surgical method of treatment that they would choose if they were the ones affected by haemorrhoidal disease.

The following questions in the survey addressed the use of antibiotics during the perioperative period, the ways the patient was prepared for the surgery, and the dietary recommendations following the treatment. After receiving the filled-in questionnaires, the answers were reviewed and data analysis was conducted.

\section{Results}

Out of the 1523 members of APS who received questionnaires, responses were received from 807 members (52.9\%).

According to the received data, it was found that a minority of surgical procedures performed for haemorrhoidal disease treatment take place in private practices (11\%). It is much more preferred to perform such procedures in an institution with an operating theatre and anaesthetic facilities (district hospitals $-46 \%$, regional hospitals or university departments $-43 \%$ ).

According to $33 \%$ of the responses, haemorrhoidectomy procedures constituted $10-20 \%$ of all proctological procedures. Twenty percent of the participants responded that $50-60 \%$ of the procedures were haemorrhoidectomies. The remaining participants reported values between $2 \%$ and $7 \%$ for haemorrhoidectomies from the total number of proctological procedures performed.

The next question concerned the age group of the patients treated for haemorrhoidal disease. The age group 30-40 years old was indicated by $16 \%$ of respondents, $40-50$ years old was indicated by $28 \%$ of respondents, $50-60$ years old was indicated by $40 \%$ of respondents, and $60-70$ years old by $6 \%$ of participants.

Answering the question about the number of patients annually treated conservatively for haemorrhoidal disease, the majority of participants (79\%) chose the answer fewer than 500 persons. Nineteen percent of participants claimed that they treated between 500 and 1000 patients conservatively, $2 \%$ of participants indicated between 1000 to 2000 as treated conservatively due to haemorrhoidal disease.

Regarding the question about the number of patients surgically treated due to haemorrhoidal disease, the majority of participants $(66 \%)$ indicated fewer than 50 patients annually. Twenty-nine percent of participants indicated an between 50 and 100 patients, and $5 \%$ of participants indicated between 100 and 200 patients.

Regarding the question about the number of life-threatening postsurgical complications of haemorrhoidal disease treatments in the last year, the vast majority of participants (93\%) indicated between 0 and 1 life threatening complications, $5 \%$ of the participants indicated 2 to 3 , and $2 \%$ of participants indicated 4 or more.

$72.5 \%$ of participants indicated the Milligan-Morgan technique as a leading surgical treatment for symptomatic haemorrhoidal disease, and Ligasure technique was indicated by $15.5 \%$ of participants. The least commonly used surgical methods of treatment of haemorrhoidal disease included: the Ferguson technique (3.5\%), DGHL 
(3.5\%), other methods (3.5\%), the Parks technique (1.7\%), and the Longo technique (0\%) (Table I).

As the least patient-friendly procedure for the treatment of haemorrhoidal disease $41.5 \%$ of participants indicated the stapler technique, $17 \%$ chose the Milligan-Morgan technique, $12 \%$ of participants chose the Ferguson technique, 10\% the Ligasure method, 3.5\% chose the Parks technique, and $2 \%$ indicated the DGHL technique. Fourteen percent of participants indicated a different method from the ones mentioned above as the least patient friendly.

For the question regarding the reason for the rare use of the Longo technique, $14 \%$ of participants indicated low financial compensation for the procedure by the National Health System, 19\% of participants indicated the high cost of the stapler as the main reason, 39.5\% of participants indicated concern with known serious complications associated with the use of Longo method, and $27.5 \%$ expressed a lack of conviction that the Lango technique would be beneficial to their patients.

When asked about what type of procedure they would chose if they themselves were affected by haemorrhoidal disease: $65 \%$ of participants responded that the Milligan-Morgan technique would be their treatment of choice, $12 \%$ of participants would choose the Fergusson technique as their preferred method, and $2 \%$ would choose the stapler method. The Parks technique was also indicated as the preferred treatment by $2 \%$ of participants, and $5 \%$ chose the Ligasure technique. None of the participants chose the DGHL procedure, while 14\% of the participating members of ASP would pick an operation different from the ones mentioned above.

When asked about the perioperative antibiotic prophylaxis $83 \%$ of participants stated using a single dose of antibiotics prior to surgery, $8.5 \%$ of participants used antibiotics also for a few days after surgery, and $8.5 \%$ used no antibiotic prophylaxis.

The vast majority of participants (70\%) used an enema in the evening of the day before the operation, as well as in the morning before the procedure, as a method of preparing the patient for surgery, $21 \%$ indicated using an enema only in the morning on the day of the procedure, $7 \%$ of participants used a mechanical method to flush the intestines, and $2 \%$ of participants used no prior preparation before the treatment.

Surgeons were asked about recommended postoperative diet. The responses were as follows: $62 \%$ of participating members of APS recommend to the patients staying on a special diet for 1 week, $24 \%$ recommend a special diet for 1 to 2 days, and $12 \%$ of participants suggested a special diet for 2 weeks. The remaining $2 \%$ of participants recommend a diet for an even longer period of time: 3 weeks or more.
Table I. Surgical treatment of choice according to surgeons.

\begin{tabular}{lc} 
Type of surgery & Surgical procedure as a method of choice (\%) \\
\hline Milligan-Morgan & 72.5 \\
\hline Ferguson & 3.5 \\
\hline Stapler (Longo) & 0 \\
\hline Parks & 1.7 \\
\hline DGHL & 3.5 \\
\hline Ligasure & 15.5 \\
\hline Other methods & 3.5
\end{tabular}

\section{Discussion}

Haemorrhoidal disease affects people of all ages. Treatment varies and depends mainly on the surgeon's preference and experience. In Poland postoperative bleeding is the main and the most serious complication. This complication is also considered to be very serious in France; however, French doctors additionally pay close attention to relieving the postoperative pain [8].

Cerato et al. showed in their meta-analysis that haemorrhoidal excision is the most commonly used method of treatment, which is also associated with less postoperative pain [9]. Similarly, in Poland the Milligan-Morgan technique is the method of choice.

Polish doctors are not fully convinced by the stapler method for the treatment of haemorrhoidal disease. The situation looks exactly the opposite in other countries, where the Longo technique is commonly used with satisfying results. Wang et al., in their meta-analysis, compared the results of treatment with stapler vs. the Milligan-Morgan technique. According to their study, the Longo technique may be successfully used, allowing for a shorter hospital stay, less postoperative pain, and shorter convalescence period. However, the long-term results show that this method is also associated with higher risk of recurrence as well as the need to reoperate [10].

In Poland, the majority of patients with stage 3 and 4 haemorrhoidal disease are refered for surgical treatment. A different opinion about this is presented by Buntzen et al. [11]. They claim that haemorrhoidal disease in all stages can be treated with a conservative method, such as the Barron procedure and DGHAL. Only a small group of patients with symptomatic haemorrhoidal disease resistant to conservative treatment should be referred for surgical treatment.

On the other hand, Altomare et al. [12] claim that patients with stage 4 disease should be treated surgically. The previously-mentioned methods of conservative treatment are much appreciated by patients; how- 
ever, they are associated with increased risk of relapse of haemorrhoidal disease.

According to Lu et al. [13], stage 2 and 3 of haemorrhoidal disease can be effectively treated with the use of band ligation with a minimal risk of complications and no need for more invasive methods of surgical treatment. This statement seems to be correct because in everyday practice many patients present with symptoms in the second and third stages of haemorrhoidal disease. It seems reasonable to begin the treatment with the use of conservative methods - including the rubber band ligation - before more invasive means of surgery are used. In many cases such conservative methods seem to be sufficient to relieve the pain and symptoms in patients with haemorrhoidal disease.

In Poland the second most commonly used method of surgical treatment of haemorrhoidal disease is the Ligasure technique. The results of treatment are comparable to those with Milligan-Morgan technique. The only problem seems to be the high cost of the single-use tip in comparison to the costs that are covered by the insurer.

Stapler costs in the Longo method are higher than the costs for the tools used in the Ligasure technique. Yang et al. [14] compared the results of treatment using Longo technique to those of the Ligasure technique. The results of both methods were comparable. The main advantage of the Ligasure technique seems to be the lower risk of skin tags, as well as a decreased risk of haemorrhoidal prolapse in the future [15].

A similar study was published by Kim et al. [16], in which they compared the differences of surgical treatments between the stapler method and Milligan-Morgan technique over a period of 5 years. The results were almost the same; less postoperative pain was the only significant difference in patients treated with Longo technique.

\section{Conclusions}

Symptomatic haemorrhoidal disease is a common problem among women and men over 45 years of age. The treatment of haemorrhoidal disease is chosen by the presence of symptoms. There are a variety of treatment options, both conservative and surgical; however, the treatment should be selected for each patient individually. In Poland, the Milligan Morgan technique is the preferred method. The majority of procedures are performed in the regional hospitals and university departments, and less commonly in private practice. Annually, in the majority of hospitals, around 50 patients are treated surgically, and less than 500 patients are treated conservatively. Surgical procedures are very rarely associated with serious complications. The vast majority of surgeons in Poland are not convinced by the stapler technique, justifying this fact with the possibility of developing serious complications. Prior to the various procedures, patients in Poland are prepared with an enema and receive antibiotic prophylaxis. A solely liquid diet is recommended for a week after the surgery for a large proportion of patients.

\section{Conflict of interest}

The authors declare no conflict of interest.

\section{References}

1. Herold A, Joos A, Bussen D. Operations for hemorrhoids: indications and techniques. Chirurg 2012; 83: 1040-8.

2. Bussen D, Herold A, Bussen S. Health-related quality of life after surgical haemorrhoid treatment - results, methods and problems. Zentralbl Chir 2012; 137: 385-9.

3. Herold A. Stage-adjusted treatment for haemorrhoidal disease. Chirurg 2008; 79: 418-29.

4. Herold A. Therapy of hemorrhoidal disease. Chirurg 2006; 77: 737-47.

5. Gaj F, Trecca A. Hemorrhoids and rectal internal mucosal prolapse: one or two conditions? A national survey. Tech Coloproctol 2005; 9: 163-5.

6. Tiernan J, Hind D, Watson A, et al. The HubBLe trial: haemorrhoidal artery ligation (HAL) versus rubber band ligation (RBL) for haemorrhoids. BMC Gastroenterol 2012; 12: 153.

7. Keskimäki I, Aro S, Teperi J. Regional variation in surgical procedure rates in Finland. Scand J Soc Med 1994; 22: 132-8.

8. Vinson-Bonnet B. Hemorrhoidal surgery: new trends for daycase surgery. Presse Med 2014; 43: 297-300.

9. Cerato MM, Cerato NL, Passos P, et al. Surgical treatment of hemorrhoids: a critical appraisal of the current options. Arq Bras Cir Dig 2014; 27: 66-70.

10. Wang GQ, Liu Y, Liu Q, et el. A meta-analysis on short and long term efficacy and safety of procedure for prolapse and hemorrhoids. Zhonghua Wai Ke Za Zhi 2013; 51: 1034-8.

11. Buntzen S, Christensen P, Khalid A, et al. Danish Surgical Society. Diagnosis and treatment of haemorrhoids. Dan Med J 2013; 60: B4754.

12. Altomare DF, Giuratrabocchetta S. Conservative and surgical treatment of haemorrhoids. Nat Rev Gastroenterol Hepatol 2013; 10: 513-21.

13. Lu LY, Zhu Y, Sun Q. A retrospective analysis of short and long term efficacy of RBL for haemorrhoids. Eur Rev Med Pharmacol Sci 2013; 17: 2827-30.

14. Yang J, Cui PJ, Han HZ, et al. Meta-analysis of stapled hemorrhoidopexy vs. LigaSure hemorrhoidectomy. World I Gastroenterol 2013; 19: 4799-807.

15. Michalik M, Pawlak M, Bobowicz M, Witzling M. Long-term outcomes of stapled hemorrhoidopexy. Videosurgery Miniinv 2014; 9: 18-23.

16. Kim JS, Vashist YK, Thieltges S, et al. Stapled hemorrhoidopexy versus Milligan-Morgan hemorrhoidectomy in circumferential third-degree hemorrhoids: long-term results of a randomized controlled trial. J Gastrointest Surg 2013; 17: 1292-8.

Received: 13.04 .2015

Accepted: 5.05 .2015 